\title{
Assessment of autonomic dysreflexia in patients with spinal cord injury
}

\author{
Armin Curt, Boris Nitsche, Biljana Rodic, Brigitte Schurch, Volker Dietz
}

\begin{abstract}
Objectives and methods-To assess the impairment of supraspinal control over spinal sympathetic centres and the occurrence of autonomic dysreflexia in patients with spinal cord injury. Autonomic dysreflexia is caused by the disconnection of spinal sympathetic centres from supraspinal control and is characterised by paroxysmal hypertensive episodes caused by non-specific stimuli below the level of the lesion. Therefore, patients with spinal cord injury were examined clinically and by different techniques to assess the occurrence of autonomic dysreflexia and to relate disturbances of the sympathetic nervous system to episodes of autonomic dysreflexia.
\end{abstract}

Results-None of the paraplegic patients, but $59 \%(13 / 22)$ of tetraplegic patients (91\% of the complete, $27 \%$ of the incomplete patients) presented signs of autonomic dysreflexia during urodynamic examination. Only $62 \%$ of the tetraplegic patients complained about symptoms of autonomic dysreflexia. Pathological sympathetic skin responses (SSRs) of the hands were related to signs of autonomic dysreflexia in $93 \%$ of cases. No patient with preserved SSR potentials of the hands and feet showed signs of autonomic dysreflexia, either clinically or during urodynamic examination. Ambulatory blood pressure measurements (ABPMs) indicated a loss of circadian blood pressure rhythm (sympathetic control) but preserved heart rate rhythm (parasympathetic regulation) only in patients with complete tetraplegia. Pathological ABPM recordings were seen in $70 \%$ of patients with symptoms of autonomic dysreflexia. Conclusions-The urodynamic examination was more sensitive in indicating signs of autonomic dysreflexia in patients with spinal cord injury, whereas SSR allowed the assessment of the degree of disconnection of the sympathetic spinal centres from supraspinal control. Using ABPM recordings the occurrence of episodes of autonomic dysreflexia over 24 hours and the effectiveness of therapeutical treatment can be assessed.
Keywords: spinal cord injury; autonomic dysreflexia; urodynamic examination; 24 hour ambulant blood pressure measurement; sympathetic skin response

In patients with spinal cord injury both the spinal somatic (motor-sensory) and autonomic (sympathetic) nervous system can be affected. ${ }^{1}$ Therefore, patients can have sympathetic failure (for example, orthostatis) due to a disconnection of the spinal sympathetic centres from supraspinal control (hypothalamus, brainstem). ${ }^{23}$ By contrast with the spinal sympathetic pathway, parasympathetic nerve fibres innervating the cardiovascular system are conveyed by the vagus nerve and run outside the spinal cord. ${ }^{4}$ Therefore, patients with spinal cord injury usually have autonomic failure due to the disconnection of the spinal sympathetic system whereas cardiac parasympathic pathways remain unaffected. ${ }^{56}$

Autonomic failure can lead to autonomic dysreflexia characterised by paroxysmal hypertensive episodes combined with headache, profuse sweating, and concurrent bradycardia. $^{7.9}$ Due to the loss of supraspinal sympathetic control, non-specific stimuli below the level of injury (for example, bladder distension, rectal manipulation, painful stimuli to the skin) can induce excessive sympathetic spinal outflow below the level of lesion, which causes the cardiovascular changes. ${ }^{1011}$ This sympathetic hyperactivity is balanced by activation of the vagus nerve via the baroreceptor reflex, which leads to the concurrent bradycardia. ${ }^{12-13}$ An adequate diagnosis and management of autonomic dysreflexia is critical for the rehabilitation programme of patients with spinal cord injury, as these episodes are potentially fatal. ${ }^{14}$ Those patients with a level of injury higher than T6 are particularly prone to autonomic dysreflexia. Therefore, this study focused on improving the diagnostic assessment of the spinal sympathetic nervous system and autonomic dysreflexia in patients with traumatic tetraplegia.

The aim of this study was to evaluate the effect of impaired function of the descending sympathetic spinal tract on (1) the sympathetic skin responses (SSRs); (2) the results of 24 hour ambulant blood pressure measurements (ABPMs); and (3) the frequency of occurrence of symptoms of autonomic dysreflexia during urodynamic examination. These variables would be expected to provide information related to the damage of the spinal 
sympathetic tract in patients with spinal cord injury and also to indicate the best diagnostic approach for the assessment of autonomic dysreflexia even if it is not clinically evident.

\section{Patients and methods}

Patients with chronic ( $>6$ months posttrauma) paraplegia and tetraplegia due to traumatic spinal cord injury who were admitted to the paraplegic centre between 1993 and 1995 were studied. All patients were younger than 65 and none were receiving cardiac or hypertensive treatment. Furthermore, patients were excluded if they had a history of cardiovascular diseases (coronary heart disease, arrhythmias, hypertension, or orthostatic dysregulation). All patients underwent neurological examination according to the international American Spinal Injury Association (ASIA) protocol, allowing the classification of the lesion as either complete or incomplete (any sensory or motor function in the sacral dermatomes). ${ }^{15}$ Furthermore, the ASIA motor (maximum 100 points) and sensory scores (maximum for both aesthesia and algesia 112 points) indicated the severity of the spinal cord lesion.

\section{SYMPATHETIC SKIN RESPONSE}

For the evaluation of the function of the descending spinal sympathetic tract the SSR of the right hand and foot were examined in accordance with techniques described elsewhere. ${ }^{16-20}$ The SSR was recorded using conventional EMG apparatus (Amplaid). Surface EMG disc electrodes (gold cup, Dantec) were attached to the ventral and dorsal surface of the right hand and foot. The SSR was recorded over 10 seconds and sampled at a band pass of $0.3 \mathrm{~Hz}$ to $3 \mathrm{kHz}$. Electrical stimulation was applied using single, square pulses $(0.2 \mathrm{~ms}$ duration, $10-20 \mathrm{~mA})$ to the contralateral median nerve at the wrist. To avoid pathological SSR recordings due to lesions of the afferent nerve fibres (for example, in traumatic tetraplegic patients) a second method was performed in parallel. Single, transcortical magnetic stimuli were applied (Mag Pro, Dantec, Denmark) using a flat round coil (MC 125 Coil Dantec, 1.4 Tesla) localised over $\mathrm{Cz}$ (according to the international 10-20 system). The stimulus duration was $0.1 \mathrm{~ms}$ and the intensity $80-100 \mathrm{~A} / \mu \mathrm{s}$. To prevent habituation the interstimulus interval was set at 60 seconds for both stimulation techniques. In accordance with other studies the SSR potentials were considered to be normal when they could reliably be reproduced three times by both stimulation techniques. Those potentials which were either not sufficiently reproducible or absent were classified as pathological.

TWENTY FOUR HOUR AMBULATORY BLOOD PRESSURE MEASUREMENT

The ABPM was performed with a SpaceLabs 90207 Ambulatory Blood Pressure System (SpaceLabs Medical GmbH, Kaarst) as previously described. ${ }^{21-23}$ Systolic blood pressure, diastolic blood pressure, and heart rate were measured and recorded automatically. For statistical analysis, measurements recorded every 20 minutes between 0800 and 1200 represented the day period, whereas hourly recordings from midnight to 0400 represented the night period. This definition was made for the following reasons. During defined day periods patients were involved in an intensive rehabilitation programme, usually mobilised in a wheelchair. The period of bed rest (night period) usually started at midnight and ended, depending on the state of rehabilitation, at 0400 with the next bladder emptying. Therefore, the definition of the day/night period was adapted to the particular needs of the patients in the rehabilitation programme.

\section{URODYNAMIC EXAMINATION}

Patients were examined urodynamically while in a supine position with $15^{\circ}$ pelvic obliquity. Two microtransducer catheters (No 5 and 3 radio-opaque, $8 \mathrm{~F}$ ) were used for the urethrovesical and anorectal pressure recordings respectively. ${ }^{24}$ Proper location of the transducers was controlled by image intensifier. Slow bladder filling ( $5 \mathrm{ml} / \mathrm{min})$ was performed with a $24 \%$ contrast medium (3-acetylamino-methyl5-acetylamino-2,4,6-tribenzoic acid). Drugs that could interfere with the urethrovesical function were discontinued 24 hours before examination. In all patients blood pressure and heart rate were continuously monitored throughout the examination (Ohmeda 2300 Finapress). As described elsewhere, ${ }^{25}$ the patients were expected to exhibit signs of autonomic dysreflexia, when they showed paroxysmal systolic and diastolic hypertensive episodes with concurrent bradycardia due to manipulations of the bladder (bladder distension, voiding, catheter positioning).

\section{DATA ANALYSIS}

The mean (SD) of the ABPM, systolic blood pressure, diastolic blood pressure, and heart rate examinations were calculated and the significance of any differences were determined with a two tailed, paired $t$ test. The level of significance was set at $P<0.01$.

\section{Results}

PATIENTS

Parallel studies were performed on 22 patients with chronic tetraplegia (11 complete, 11 incomplete; $18 \mathrm{men}$, four women) and nine patients with complete paraplegia (level of lesion < T6, seven men, two women). The second group served as controls, as they were not likely to develop autonomic dysreflexia. Table 1 shows the demographic and clinical data (ASIA scores).

\section{SYMPATHETIC SKIN RESPONSE}

No patient with complete tetraplegia displayed normal SSR potentials in the hands or feet using either of the stimulation techniques. In all patients with complete paraplegia SSR potentials of the hands could be recorded, but only $60 \%$ (six of 11 ) gave responses of the feet. In $73 \%$ (eight of 11 ) of the patients with 
Table 1 Demographic and clinical data of the tetraplegic and paraplegic patients studied

\begin{tabular}{|c|c|c|c|c|c|}
\hline \multirow[b]{2}{*}{ Patients } & \multirow{2}{*}{$\begin{array}{l}\text { Level of } \\
\text { injury }\end{array}$} & \multirow{2}{*}{$\begin{array}{l}\text { Age } \\
\text { mean (range) }\end{array}$} & \multicolumn{3}{|c|}{$A S I A$ scores (mean $S D$ ) } \\
\hline & & & $\operatorname{motor}(\max 100)$ & algesia (max 112) & aesthesia ( $\max 112)$ \\
\hline Complete tetraplegia & $\mathrm{C} 4-\mathrm{C} 7$ & $\begin{array}{l}29 \cdot 3 \\
(17-56)\end{array}$ & $\begin{array}{l}17 \\
(9 \cdot 2)\end{array}$ & $\begin{array}{l}20 \\
(4 \cdot 6)\end{array}$ & $\begin{array}{l}24 \\
(15)\end{array}$ \\
\hline Incomplete tetraplegia & $\mathrm{C} 2-\mathrm{C} 8$ & $40 \cdot 1$ & 80 & 91 & 91 \\
\hline $\begin{array}{l}\text { Complete paraplegia } \\
(n=9)\end{array}$ & T6-L4 & $\begin{array}{l}33 \cdot 4 \\
(19-50)\end{array}$ & $\begin{array}{l}52 \\
(3.9)\end{array}$ & $\begin{array}{l}65 \\
(17)\end{array}$ & $\begin{array}{l}68 \\
(18)\end{array}$ \\
\hline
\end{tabular}

incomplete tetraplegia (as defined by the ASIA protocol which focuses on somatic nerve function) normal SSR potentials could be recorded in both the hands and the feet (fig 1). Therefore, the clinical ASIA classification failed to accurately assess the extent of disconnection of the spinal sympathetic system in $27 \%$ (three of 11 ) of patients within the group with incomplete spinal cord injury.

No patient with preserved SSR potentials of the hands and feet showed symptoms of autonomic dysreflexia either clinically or during urodynamic examination. Conversely, all patients with symptoms of autonomic dysreflexia showed pathological SSR potentials of the hands and feet. Loss of SSR potentials of the hands was associated with symptoms of autonomic dysreflexia in $93 \%$ ( 12 of 13) of the patients.

\section{TWENTY FOUR HOUR AMBULATORY BLOOD} PRESSURE MEASUREMENT

All patients with incomplete tetraplegia $(\mathrm{n}=$ 20 recordings) and the patients with complete paraplegia ( $\mathrm{n}=10$ recordings) showed normal circadian rhythmicity of blood pressure (fig 2A). The mean systolic blood pressure during the day period was $118.2(13.2) \mathrm{mm}$ $\mathrm{Hg}$ with a significant reduction during the night period to $109.1 \quad(8.4) \mathrm{mm} \mathrm{Hg}$ ( $\mathrm{P}<$ $0.001)$. The same was true for the diastolic

Figure 1 (A) Normal sympathetic skin response of the hands (upper traces) of the hands (upper traces) and feet (lower traces) of a
healthy subject $(H)$. (B) Loss of the response in a patient with complete tetraplegia (P).

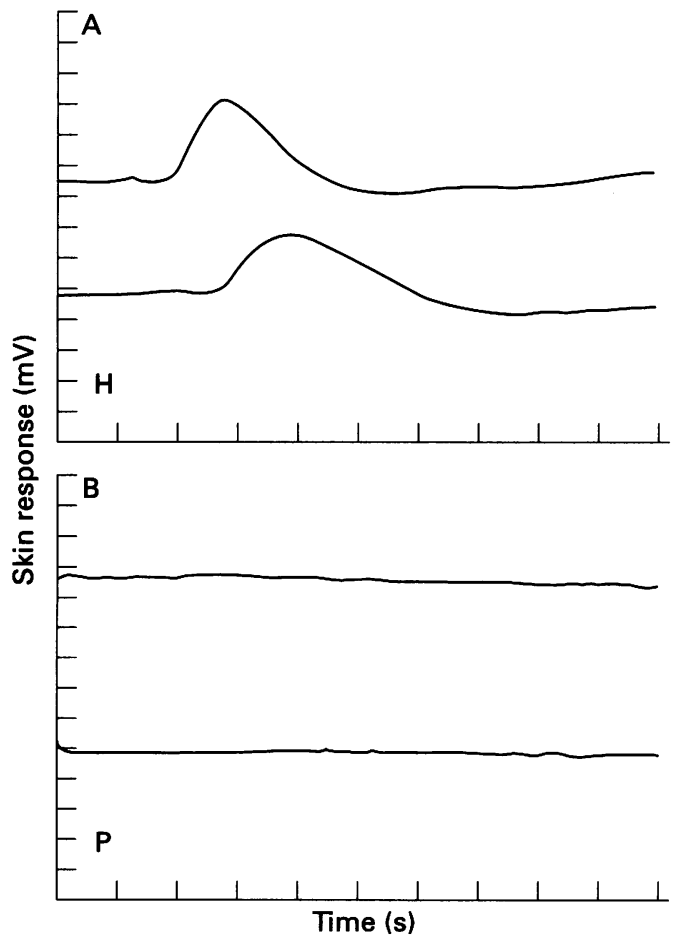

blood pressure with reduction from 72.5 (9.7) $\mathrm{mm} \mathrm{Hg}$ to 62.9 (6.5) $\mathrm{mm} \mathrm{Hg}$ (P < 0.0001 ). In $91 \%$ (10 of 11 ) of patients with complete tetraplegia ( $\mathbf{n}=32$ recordings) the physiological rhythmicity of circadian blood pressure was absent (fig $2 B, C$ ). There was no significant difference between the values of the day period (systolic blood pressure 109.7 (8.5) $\mathrm{mm} \mathrm{Hg}$; diastolic blood pressure 62.6 $(7.5) \mathrm{mm} \mathrm{Hg}$ ) and those of the night period (systolic blood pressure $113.6(9.4) \mathrm{mm} \mathrm{Hg}$; diastolic blood pressure $63 \cdot 1(8 \cdot 1) \mathrm{mm} \mathrm{Hg}$ ).

Two patients with complete tetraplegia with symptoms of autonomic dysreflexia and episodes of severe cardiovascular dysregulation (paroxysmal hypertensive episodes up to $224 \mathrm{~mm} \mathrm{Hg}$ with concurrent bradycardia up to $46 \mathrm{bpm}$ ) disclosed a pathological ABPM profile reflecting these episodes (fig 2B). After effective treatment (antihypertensive drugs, sphincterotomy) the ABPM profile showed the characteristics of patients with complete tetraplegia (loss of circadian rhythmicity) without the episodes of severe blood pressure dysregulation (fig 2C).

By contrast with the blood pressure recordings, the circadian rhythmicity of the heart rate was preserved in all tetraplegic and paraplegic patients. The patients with complete tetraplegia (heart rate $71 \cdot 1(17 \cdot 7)$ to $60 \cdot 4(11 \cdot 4) \mathrm{bpm}$ ) as well as the patients with incomplete tetraplegia (heart rate $83.2(13.2)$ to 68.1 $(10.6) \mathrm{bpm})$ showed a significant $(P<0.001)$ reduction in heart rate during the night period. Therefore, the results of ABPM showed severe dysfunction of the spinal sympathetic system in patients with complete tetraplegia, whereas the parasympathetic regulation was normal (because the circadian rhythmicity in heart rate is controlled by the extraspinal vagal nerve).

Of those patients presenting symptoms of autonomic dysreflexia $70 \%$ (nine of 13) exhibited pathological ABPM recordings. The loss of circadian rhythmicity of blood pressure was associated with the occurrence of autonomic dysreflexia in $91 \%$ (12 of 13 ) of patients. Of those patients with pathological SSR potentials $27 \%$ (three of 11 ) still showed normal ABPM measurements.

\section{URODYNAMIC EXAMINATION}

During urodynamic examination, which was combined with continous monitoring of the blood pressure and heart rate, no paraplegic patient (level of spinal cord injury < T6), but $59 \%$ (13 of 22) of the tetraplegic patients displayed signs of autonomic dysreflexia. Of the patients with clinically complete tetraplegia 

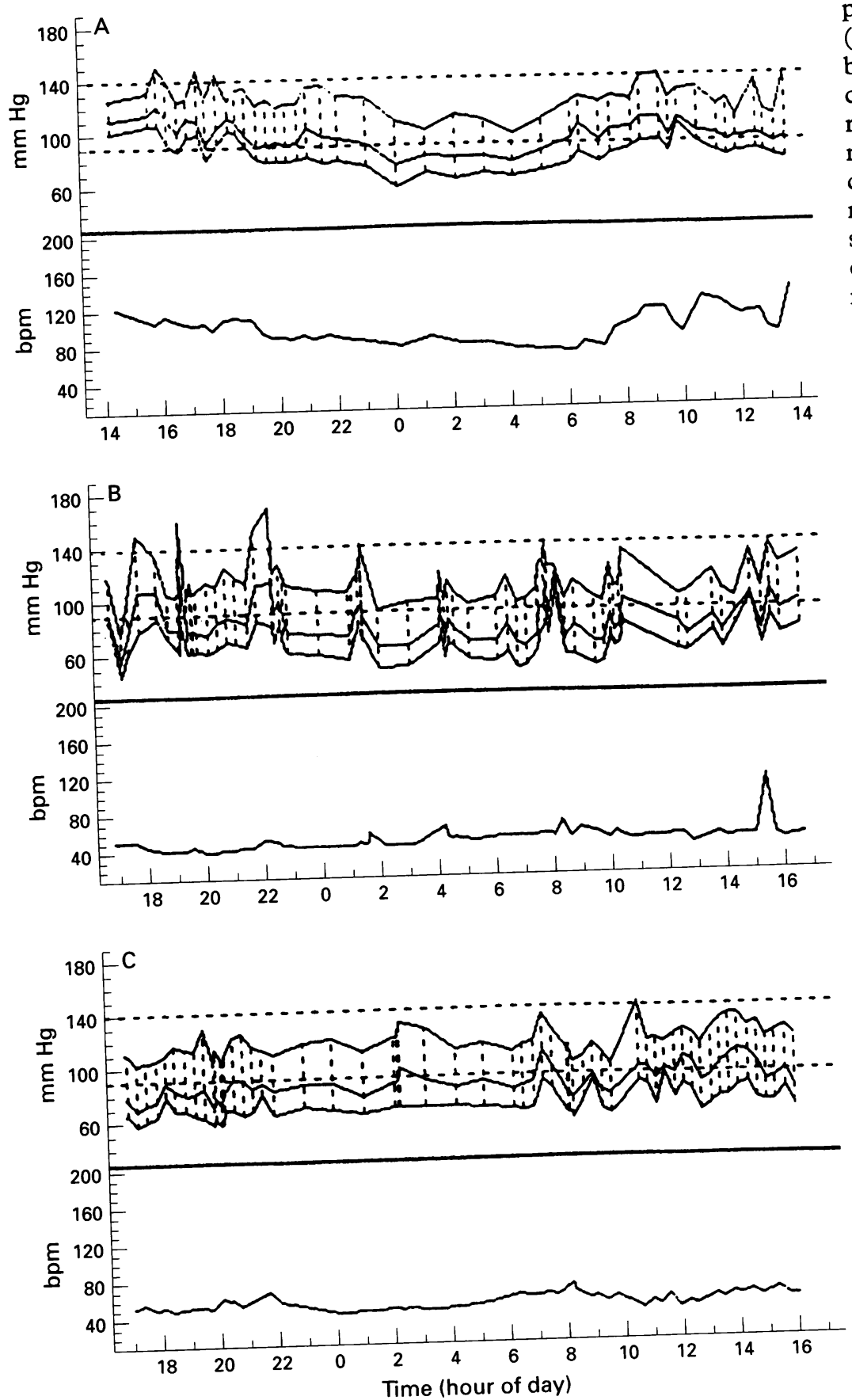

Figure 2 (A) Normal ABPM measurements with a preserved physiological drop of blood Figure 2 (A) Normal ABPM measurements with a preserved physiological a patient with pressure during the night period (midnight to 0400). (B) Recordings from Recordings from complete tetraplegia and multiple episodes of autonomic dysreflexia. (C) Recor

the same patient after effective treatment of autonomic dysreflexia with the antihypertensi blood pressure.

(ASIA classification) $91 \%$ (10 of 11 ) and of those with incomplete tetraplegia $27 \%$ (three of 11 ) showed signs of autonomic dysreflexia. During urodynamic examination these

Table 2 Changes in systolic (SBP) and dystolic (DBP) blood pressures and heart rate THR in patients with signs of autonomic dysreflexia during urodynamic examination

\begin{tabular}{|c|c|c|c|c|}
\hline Cardiovascular values & $\begin{array}{l}\text { Basic } \\
\text { mean (SD) }\end{array}$ & $\begin{array}{l}\text { Dysreflexia } \\
\text { mean }(S D)\end{array}$ & Difference (\%) & $\begin{array}{l}\text { Absolute } \\
\text { difference }\end{array}$ \\
\hline $\begin{array}{l}\text { SBP }(\mathrm{mm} \mathrm{Hg}) \\
\text { DBP (mm Hg) } \\
\text { HR (bpm) }\end{array}$ & $\begin{array}{c}115(17) \\
64(13) \\
59(6)\end{array}$ & $\begin{array}{r}155(32) \\
82(16) \\
53(10)\end{array}$ & $\begin{array}{r}35 \cdot 5 \\
31 \cdot 2 \\
-14.3\end{array}$ & $\begin{array}{c}40(25) \\
17(16) \\
8(6)\end{array}$ \\
\hline
\end{tabular}

$\mathrm{P}<0.01$. patients showed episodes with significant $(\mathbf{P}<0.001)$ increases of systolic and diastolic blood pressures of over $30 \%$ (table 2 ) and a concurrent significant reduction of the heart rate of about $15 \%(P<0.01)$. Signs of autonomic dysreflexia were induced by bladder distension and were related mainly to hyperreflexive bladder contractions and detrusorsphincter dyssynergia. Only $62 \%$ (eight of 13 ) of the patients had clinical symptoms of autonomic dysreflexia.

\section{Discussion}

Tetraplegic patients and patients with a high level of thoracic spinal cord injury $(>\mathrm{T} 6)$ are prone to develop autonomic dysreflexia, which is an autonomic failure due to the loss of supraspinal control of spinal sympathetic centres below the level of lesion. ${ }^{26}$ After recovering from spinal shock the remaining sympathetic spinal centres can become hyperreflexive such that non-specific stimuli below the level of lesion can provoke uncontrolled, excessive sympathetic outflow with subsequent cardiovascular effects. ${ }^{4}$ The most effective stimulus to provoke such hyperreflexive sympathetic activity below the level of lesion seems to be bladder distension. ${ }^{7}$ In patients with spinal cord injury the enhanced sympathetic activity due to bladder stimulation could be shown by recording SSR potentials during urodynamic examination. ${ }^{27}$

In the present study SSR potentials were recorded in patients with spinal cord injury to test the function of the descending spinal sympathetic tract. In accordance with studies performed in other spinal cord diseases the technique used here allows the assessment of the degree to which supraspinal control of the spinal sympathetic system is impaired..$^{28}{ }^{29}$ The findings show that a loss of or impaired spinal sympathetic control is related to the development of autonomic dysreflexia. No patient with preserved SSR potentials showed autonomic dysreflexia symptoms either clinically or during urodynamic examination. By contrast, all patients with autonomic dysreflexia symptoms had pathological SSR. This fits in with the finding that in three tetraplegic patients with incomplete lesions of the somatic nervous system (motor and sensory functions), but complete disconnection of the autonomic spinal centres (abolished SSR potentials after supralesional stimulation) displayed typical signs of autonomic dysreflexia during urodynamic examination. Therefore, autonomic failure can be assessed by the recording of SSR potentials, even when they are not clinically evident.

The influence of the descending spinal sympathetic tract on the cardiovascular system can also be assessed using ABPM recordings. ${ }^{26} \mathrm{By}$ contrast with healthy subjects and both paraplegic and patients with incomplete tetraplegia, almost all patients with complete tetraplegia showed a loss of physiological regulation of circadian blood pressure. This is consistent with findings from patients with pure autonomic failure, who also show a loss of cir- 
cadian rhythmicity of blood pressure. ${ }^{30} \mathrm{By}$ contrast with the impaired circadian rhythmicity of blood pressure in patients with complete tetraplegia, the regulation of the circadian heart rate rhythmicity was preserved. This is due to the fact that the this regulation is under the control of the vagus nerve. ${ }^{12}$

Nevertheless one third of patients lacking SSR had a preserved rhythmicity of physiological circadian blood pressure and only about two thirds of patients with typical signs of autonomic dysreflexia during urodynamic examination showed a loss of the physiological circadian blood pressure regulation. These findings indicate that blood pressure regulation is more resistant to impairment after disconnection of the spinal sympathetic centres. However, the ABPM recordings are useful in recording the frequency and severity of episodes of autonomic dysreflexia during daily living. Furthermore, the effectiveness of medical (antihypertensive drugs) and surgical (sphincterotomy) treatment of these patients can be monitored using ABPM recordings. ${ }^{21}$

Urodynamic examination can be regarded as an effective and standardised diagnostic procedure for evoking signs of autonomic dysreflexia. In this study it was clear that urodynamic examination is a very sensitive tool in the diagnosis of autonomic dysreflexia, as only half of the patients who showed signs of autonomic dysreflexia during urodynamic examination presented clinical symptoms of autonomic dysreflexia. As described earlier ${ }^{25} 31$ episodes of autonomic dysreflexia during urodynamic examination were characterised by increases of both systolic and diastolic blood pressures by about $30 \%$, while the heart rate decreased by about $15 \%$. Therefore, the recording of cardiovascular variables during urodynamic examinations is important to assess signs of autonomic dysreflexia.

\section{Conclusion}

In patients with spinal cord injury autonomic failure due to the impaired function of the spinal sympathetic tract can be assessed by different techniques. The SSR recordings are the most sensitive at indicating the degree of damage of the descending spinal sympathetic system. The combination of urodynamic examinations and ABPM recordings allows the prediction of the occurrence and severity of autonomic failure (sympathetic disconnection). Therefore, early diagnosis and treatment of autonomic dysreflexia, one of the most serious complications in patients with a high level (> T6) of spinal cord injury, becomes possible. This work was supported by grants from the Swiss National
Science Foundation (No $31-42899.95)$ and the International Research Institute for Paraplegia (P16/93).

1 Kerr FW, Alexander S. Descending autonomic pathways in the spinal cord. Arch Neurol 1964;10:249-61.
2 Guttmann L, Munro AF, Robinson R, Walsh JJ. Effect of tilting on the cardiovascular responses and plasma catecholamine levels in spinal man. Paraplegia 1963;1:4-19.

3 Engel P, Hildebrandt G. Long-term studies about orthostatic training after high spinal cord injury. Paraplegia 1976;14:159-64.

4 Mathias CJ, Christensen NJ, Frankel HL, Spalding JMK. Cardiovascular control in recently injured tetraplegics in spinal shock. Quarterly fournal of Medicine New Series XLVIII 1979;190:273-87.

5 Fuhrer MJ. Skin conductance responses mediated by the transected human spinal cord. $\mathscr{F}$ Appl Physiol 1971;30: transected

6 Stjernberg L, Blumberg H, Wallin G. Sympathetic activity in man after spinal cord injury. Brain 1986;109:695-715.

7 Guttmann L, Whitteridge D. Effects of bladder distension on autonomic mechanism after spinal cord injury. Brain 1947;70:361-404

8 Krum H, Howes LG, Brown DL, Louis WJ. Blood pressure variability in tetraplegic patients with autonomic hyperreflexia. Paraplegia 1989;27:284-8.

9 Head H, Riddock G. The automatic bladder, excessive and some other reflex conditions in gross injuries of the spinal cord. Brain 1917;40:188-263.

10 Nanninga JB, Rosen JS, Krumlovsky F. Effect of autonomic hyperreflexia on plasma renin. Urology 1976;6: 638-40.

11 Wallin BG, Stjernberg L. Sympathetic activity in man after spinal cord injury. Brain 1984;107:183-98.

12 Reis DJ, Nathan MA, Doba N. Two specific brainstem systems which regulate the blood pressure. Clin Exp tems which regulate the blood press
Pharmacol Physiol Suppl 1975;2:179-83.

13 Mathias CJ, Frankel HL, Christensen NJ, Spalding JMK. Enhanced pressor response to noradrenaline in patients with cervical spinal cord transection. Brain 1976;99: 757-70

14 Eltorai I, Kim R, Vulpe M, Kasravi H, Ho W. Fatal cerebral hemorrhage due to autonomic dysreflexia in a tetraplegic patient: case report and review. Paraplegia 1992;30:355-60.

15 Ditunno JF, Young W, Donovan WH, Creasey G. The international standards booklet for neurological and functional classification of spinal cord injury. Paraplegia 1994;32:70-80.

16 Fagius J, Wallin BG. Sympathetic reflex latencies and conduction velocities in normal man. $f$ Neurol Sci 1980 ; 47:433-48.

17 Knezevic W, Bajada S. Peripheral autonomic surface potential: a quantitative technique for recording sympapotential: a quantitative technique for recording sympa-

18 Matsunaga K, Uozumi T, Tsuji S, Murai Y. Sympathetic skin responses evoked by magnetic stimulation of the neck. F Neurol Sci 1995;128:188-94.

19 Rossini PM, Massa R, Sancesario G, Boccasena P. Sudomotor skin responses to brain stimulation do not depend on nerve sensory fiber functionality. Electroencephalogr Clin Neurophysiol 1993;89:447-51.

20 Uozumi T, Nakano S, Matsunaga K, Tsuji S, Murai Y. Sudomotor potential evoked by magnetic stimulation of the neck. Neurology 1993;43:1397-400.

21 Chau NP, Bauduceau B, Chanudet X, Lauroque $P$, Gautier D. Ambulatory blood pressure in diabetic subjects. Am $\mathcal{F}$ Hypertens 1994;7:487-91.

22 Hsu CY. Orthostatic hypotension with brainstem tumors. Neurology 1984;34:1137-43.

23 Sivieri R, Deandrea M, Gai V, Cavallo-Perin P. Circadian blood pressure levels in normotensive, normoalbuminuric type 1 diabetic patients. Diabet Med 1994;11 357-61.

24 Rossier AB, Fam BA. 5-microtransducer catheter in evaluation of neurogenic bladder function. Urology 1986;17: 371-8.

25 Krum H, Louis WJ, Brown DJ, Clarke SJ, Fleming JA, Howes LG. Cardiovascular and vasoactive hormone responses to bladder distension in spinal and normal man. Paraplegia 1992;30:348-54.

26 Mathias CJ, Frankel HL. Clinical manifestations of malfunctioning symapthetic mechanisms in tetraplegic man. $\mathcal{f}$ Auton Nerv Syst 1983;7:303-12.

27 Prèvinaire JG, Soler JM, Hanson P. Skin potential recordings during cystometry in spinal cord injured patients. International Medical Society for Paraplegia 1993;31: Internation.

28 Yokota T, Matsunaga T, Okiyama R, Hirose $\mathrm{K}$, Tanabe $\mathrm{H}$ Furukawa T, Tsukagoshi H. Sympathetic skin response in patients with multiple sclerosis compared with patients with spinal cord transection and normal controls. Brain 1991;114:1381-94.

29 Dettmers C, Fatepour D, Faust H, Jerusalem F. Sympathetic skin response abnormalities in amyotrophic lateral sclerosis. Muscle Nerve 1993;16:930-4.

30 Mann S, Altmann DG, Raferty EB, Bannister R. Circadian variation of blood pressure in autonomic failure. Circulation 1983;68:477-83.

31 Fagius J, Karhuvaara S. Sympathetic activity and blood pressure increases with bladder distension in humans. Hypertension 1989;14:511-7. 